\title{
Prevalencia de anticuerpos IgG contra Toxoplasma gondii en donantes de sangre cubanos.
}

\section{Comunicación Breve}

Ivonne Martín-Hernández ${ }^{1}$, Susana M. García-Izquierdo ${ }^{2}$.

${ }^{1}$ Laboratorio de Errores Innatos del Metabolismo. Centro Nacional de Genética Médica. ${ }^{2}$ TecnoSUMA Internacional. Centro de Inmunoensayo, Habana, Cuba.

\section{RESUMEN.}

Introducción. La toxoplasmosis es una infección humana extendida mundialmente y su frecuencia varía mucho según las zonas geográficas y los hábitos alimentarios. En Cuba esta infección es frecuente, aunque la presencia de anticuerpos específicos varía entre 50-75\% en relación al área geográfica analizada y especialmente de la técnica empleada.

Materiales y métodos. Se realizó un estudio seroepidemiológico en 922 donantes de sangre del Banco de sangre del Municipio Marianao, Ciudad de la Habana, con la finalidad de conocer la prevalencia de anticuerpos IgG anti-Toxoplasma gondii, sus concentraciones expresadas en $\mathrm{UI} / \mathrm{mL}$ y el comportamiento de la seropositividad con la edad. Fue utilizado el ensayo inmunoenzimático heterogéneo de tipo indirecto UMELISA Toxoplasma ${ }^{a}$, para la detección de anticuerpos de tipo $\operatorname{IgG}$ contra Toxoplasma gondii en muestras de suero.

Resultados. Se encontró una seroprevalencia contra Toxoplasma gondii de 73,43\%, además de un incremento de la seropositividad con la edad y una disminución de los porcentajes de reactividad en la medida que aumentan los títulos. La presencia de anticuerpos IgG específicos se comportó de manera similar para los grupos etáreos de 20-24, 25-29 y 30-34 años, no encontrándose diferencias significativas entre ellos ( $p>0,05)$. Así mismo, éstos difirieron significativamente $(\mathrm{p}<0,05)$ con las edades de 35-39 y de 40 ó más años, pero no difirieron entre sí.

Discusión. La alta prevalencia de anticuerpos IgG anti-Toxoplasma gondii nos conduce a considerar a esta provincia como una zona de endemia intensa.

(Rev Biomed 2003; 14:247-251)

Palabras clave: Toxoplasma gondii, anticuerpos IgG, UMELISA, donantes de sangre.

\section{SUMMARY. \\ Prevalence of IgG antibodies against Toxoplasma gondii in Cuban blood donors.}

Solicitud de sobretiros: Lic. Ivonne Martín Hernández, Centro Nacional de Genética Médica, Centro colaborador de la OMS para el desarrollo de enfoques genéticos en la promoción de salud. Calle 146 No. 3102 esq. ave 31. Marianao C.P. 11600. Ciudad de la Habana. Cuba. E-mail: ivonne@infomed.sld.cu

Recibido el 25/Junio/2003. Aceptado para publicación el 19/Septiembre/2003.

Este artículo está disponible en http://www.uady.mx/sitios/biomedic/revbiomed/pdf/rb031445.pdf

Vol.14/No.4/Octubre-Diciembre, 2003 


\section{Martín-Hernández, SM García-Izquierdo.}

Introduction. Toxoplasmosis is a human infection disease that occurs worldwide and its frequency varies among different geographical zones and eating habits. In Cuba this infection is common, although the presence of specific antibodies varies between 50$75 \%$ in relation with the geographical area and the detection method employed.

Materials and methods. A seroepidemiological study of 922 blood donors' serum samples from the Municipality of Marianao, Havana City, was carried out with the purpose of measuring concentrations of anti-T. gondii $\mathrm{IgG}$ antibodies (UI/mL) and the behaviour of seropositivity with age. The heterogeneous immunoenzimatic assay UMELISA Toxoplasma $^{\text {a }}$ was used for the detection of $\operatorname{IgG}$ antibodies to Toxoplasma gondii in serum samples. Results. A seroprevalence to Toxoplasma gondii of $73,43 \%$ was found, additionally an increase in seropositivity with age and a decrease of reactivity percentages with the increase of titles were observed. The presence of specific IgG antibodies showed similar behaviour for the age groups 20-24, 25-29, and 3034 years old with no significant difference between them $(p>0,05)$. In the same way, they differ significantly $(\mathrm{p}<0,05)$ from to 35-39 and 40-or more year old age groups, although these age groups showed no difference between each other.

Discussion. The high prevalence of anti-Toxoplasma gondii IgG antibodies in the Municipality of Marianao lead us to consider this province as an intensive endemic zone. (Rev Biomed 2003; 14:247-251)

Key words: Toxoplasma gondii, IgG antibodies, UMELISA, blood donors.

\section{INTRODUCCIÓN.}

La toxoplasmosis es una infección humana extendida por todo el mundo y su frecuencia varía según las zonas geográficas y los hábitos alimentarios (1). Estudios realizados en Cuba en la década de los años 70 encontraron una prevalencia a la infección por Toxoplasma gondii (T. gondii) entre $25 \%$ y $30 \%$. En la actualidad, esta parasitosis es frecuente y, a juzgar por el número de informes, la presencia de anticuerpos específicos varía entre $50 \%$ y $75 \%$ de acuerdo al área geográfica analizada y especialmente del inmunodiagnóstico empleado para obtener estos datos (2-9).

Este trabajo, realizado mediante la técnica serológica UMELISA Toxoplasma ${ }^{a}$, da a conocer la seroprevalencia de esta parasitosis en donantes de sangre de la capital de Cuba y su relación en función de la edad, contribuyendo al conocimiento de la epidemiología de la toxoplasmosis en Latinoamérica.

\section{MATERIAL Y MÉTODOS.}

En el primer semestre del año 2000 se colectaron un total de 922 muestras de suero en una población sin datos clínicos previos o actuales de toxoplasmosis. Las muestras procedían del Banco de sangre del Municipio Marianao de la Ciudad de la Habana. Dichas muestras resultaron negativas a anticuerpos contra el virus de la inmunodeficiencia humana, al antígeno de superficie de la hepatitis y a la prueba de sífilis (VDRL).

Las muestras de sangre se obtuvieron según las técnicas convencionales de venopunción y posteriormente los sueros se separaron por centrifugación. Se colectaron en viales y se guardaron a $-20^{\circ} \mathrm{C}$ durante un tiempo no mayor de dos meses antes de ser procesados en busca de anticuerpos IgG anti-T. gondii. En este trabajo se empleó el estuche UMELISA Toxoplasma, desarrollado y producido en el Centro de Inmunoensayo. Esta técnica es rápida, sencilla y confiable. Presenta una sensibilidad de 3 $\mathrm{UI} / \mathrm{mL}$, los coeficientes de variabilidad (CV) intraensayos se encuentran entre 1,4-8,0 \% y los CV interensayos entre 4,8-10,4\%. Su recuperación es de 83-96\%.

El UMELISA Toxoplasma $a^{a}$ es un ensayo inmunoenzimático heterogéneo de tipo indirecto, que permite cuantificar hasta $1000 \mathrm{UI} / \mathrm{mL}$ de anticuerpos IgG anti-T. gondii en muestras de suero y utiliza como fase sólida tiras de poliestireno sensibilizadas previamente con un antígeno soluble de $T$. gondii. Los sueros diluidos 1:101, si presentan anticuerpos 


\section{Prevalencia de Acs-T.gondii IgG en donantes de sangre cubanos.}

específicos, forman un inmunocomplejo que es reconocido posteriormente por un complejo anti-IgG humana obtenida en carnero y conjugada con fosfatasa alcalina. Entre un paso y otro se realizaron 4 lavados de las tiras de reacción con solución reguladora de fosfato $\mathrm{pH}$ 9,8. Las incubaciones de muestras y conjugado se realizaron durante media hora a $37^{\circ} \mathrm{C}$. El volumen de reactivos utilizados en cada paso de la técnica es $10 \mu \mathrm{L}$. Finalmente, se reveló la reacción con la adición de un sustrato fluorogénico y en los resultados la intensidad de la fluorescencia emitida es proporcional a la concentración de anticuerpos presentes en la muestra.

Los datos fueron almacenados y procesados por computadora, empleando el tabulador electrónico Microsoft Excel 2000. Se calculó la frecuencia por rango de concentración y los porcentajes de reactividad para cada rango de concentración. Para el procesamiento estadístico de los datos, agrupados en intervalos de cinco años, se empleó el método de comparación múltiple de proporciones y en el caso que existiera diferencia significativa, la docimástica de Duncan $(p<0,05)$ para conocer entre qué niveles del parámetro en estudio se manifestó esa diferencia.

\section{RESULTADOS.}

Los resultados de la búsqueda de anticuerpos contra $T$. gondii se muestran en el cuadro 1 . Están agrupados en grupos de edades con intervalos de 5 años y diferentes rangos de reactividad. De los 922 sueros evaluados, el $73,43 \%$ resultaron positivos para anticuerpos $\mathrm{IgG}$ contra $T$. gondii.

Las prevalencias de anticuerpos $\operatorname{IgG}$ a $T$. gondii en los individuos con edades de 20-24 años (38/53, $71,7 \%), 25-29$ años (274/391, 70,08\%) y de 30-34 años $(256 / 365,70,14 \%)$ no difieren entre sí $(p>0,05)$. Sin embargo, estas prevalencias difieren significativamente $(\mathrm{p}<0,05)$ con las encontradas en los grupos de edades de 35-39 años $(78 / 81,96,29 \%)$ y de 40 ó más años (31/32, 96,87\%), las cuales no difieren entre sí.

\section{DISCUSIÓN.}

La seropositividad de las poblaciones para $T$. gondii a nivel mundial, y en especial en Latinoámerica, ha sido analizada por muchos investigadores, en referencia al clima, altitud, ocupación, ambiente urbano o rural, etnicidad y hábitos individuales. La alta prevalencia de anticuerpos $\operatorname{IgG}$ contra $T$. gondii encontrada en nuestro estudio $(73,43 \%)$, nos permite inferir que la provincia Ciudad de la Habana, es una zona endémica importante para este parásito (5). Yucatán, México se encuentra considerada también zona de endemia alta para Toxoplasma reportando una seropositividad en donantes de sangre de un $69 \%$ (10). Estudios comparativos realizados en la Ciudad de Buenos Aires, Argentina en población general (hombres y mujeres sanos) señalan disminución

\section{Cuadro 1}

Resultados de la búsqueda de anticuerpos anti-Toxoplasma gondii en donantes de sangre por el UMELISA Toxoplasma.

\begin{tabular}{|c|c|c|c|c|c|c|c|}
\hline \multirow{2}{*}{$\begin{array}{l}\text { RANGO } \\
\text { DE } \\
\text { EDAD } \\
\text { (AÑOS) }\end{array}$} & \multirow{2}{*}{$\begin{array}{c}\text { No. } \\
\text { INDIV. } \\
(\%)\end{array}$} & \multicolumn{6}{|c|}{ UI/mL de anticuerpos IgG anti-T. gondii. No. Indiv. y (\%) } \\
\hline & & $\begin{array}{l}>12.5 \\
<125\end{array}$ & $\begin{array}{r}3125 \\
<250\end{array}$ & $\begin{array}{l}3250 \\
<500\end{array}$ & $\begin{array}{r}3500 \\
<1000\end{array}$ & ${ }^{3} \mathbf{1 0 0 0}$ & $\begin{array}{l}\text { No. } \\
\text { React. } \\
(\%)\end{array}$ \\
\hline $20-24$ & $53(5,75)$ & 15 & 9 & 7 & 6 & 1 & $38(71,7)$ \\
\hline $25-29$ & $391(42,41)$ & 137 & 79 & 57 & 1 & 0 & $274(70,08)$ \\
\hline $30-34$ & $365(39,59)$ & 144 & 102 & 10 & 0 & 0 & $256(70,14)$ \\
\hline $35-39$ & $81(8,79)$ & 32 & 41 & 3 & 0 & 2 & $78(96,29)$ \\
\hline 40-ó más & $32(3,47)$ & 23 & 3 & 4 & 0 & 1 & $31(96,87)$ \\
\hline
\end{tabular}




\section{Martín-Hernández, SM García-Izquierdo.}

importante de seroprevalencia desde un $67,4 \%$ en el año 1967 hasta un 39,5\% en el año 1992 (11). En algunas áreas de Brasil la prevalencia serológica de infección por T. gondii oscila en los rangos de 50 a $80 \%$ en población adulta $(12,13)$. En Costa Rica estudios serológicos revelan $76 \%$ de prevalencia de anticuerpos de clase IgG contra este parásito (14). Como se puede apreciar, en todos estos países tropicales la prevalencia es alta, concordando con lo reportado por Wallace en 1969 (15).

En Cuba, la prevalencia de infección toxoplásmica ha sido estudiada por diversos autores y mediante diferentes técnicas. Resultados semejantes a los obtenidos en este estudio (70,9\%, 71\% y 75\%), han sido reportados $(3,5,8,9)$. En cambio, los resultados de este trabajo son ligeramente más elevados que lo informado por Machín y colaboradores en 1988, quienes presentaron $69,82 \%$ de positividad a $T$. gondii para la provincia de la Habana. Posteriormente en 1992, Acosta y colaboradores empleando el UMELISA Toxoplasma ${ }^{a}$ reportaron $63 \%$ de reactividad sobre una población de 793 individuos de uno y otro sexo estudiados al azar. En 1993, López y colaboradores con el mismo estuche de reactivos, evaluaron 1036 muestras de suero de donantes de sangre y encontraron 66,28\% de reactividad $(7,16,17)$.

Influyen, a criterio nuestro, tres factores importantes que contribuyen a que en la región de estudio se haya encontrado este elevado porcentaje de reactividad. Ellos son: 1) el clima cálido y húmedo de nuestro país favorece al parásito; 2) en esta provincia, con frecuencia se encuentran gatos errantes, aumentando con ello la posibilidad de que gatos infectados viertan heces fecales contaminadas al medio; 3) al Banco de sangre de Marianao asisten muchas personas de zona urbana, pero también de zonas suburbanas y rurales.

Las diferencias de la seroprevalencia en los diferentes estudios realizados en Cuba, pudieran estar dadas por el número de muestras analizadas en cada estudio, la técnica empleada o, tal como ha sido indicado por Foulon y colaboradores $(5,18)$, el cambio en la frecuencia de la infección durante el tiempo.

En los resultados, al igual que en la literatura revisada apreciamos una marcada tendencia a la disminución gradual de los porcentajes de reactividad en la medida que aumentan los títulos. De ello podemos inferir que el contacto con el parásito ocurre de forma esporádica dejando niveles descendentes de anticuerpos, cuya detección en función del tiempo, demuestran en mayor proporción títulos bajos y estables que permanecen presentes durante largo tiempo o por contacto repetido con $T$. gondii $(5,19)$.

$\mathrm{Al}$ analizar la población de donantes por grupos de edades los resultados mostraron que la tasa de seropositividad/edad tiende al aumento, desde el $70 \%$ hasta el $96 \%$.

Al igual que en nuestro estudio, en reportes por otros investigadores, se ha encontrado que al aumentar la edad, el tiempo probable de exposición al parásito también aumenta y con ello las oportunidades de seroconversión para los sujetos negativos $(20,21)$.

Debido a la aparente escasa importancia de esta parasitosis en la salud humana, no se justifica la determinación de anticuerpos contra este parásito de manera rutinaria en personas supuestamente sanas. Sin embargo, se deben tener en cuenta las consecuencias que conlleva la ruptura del equilibrio hospedero-parásito debido a deficiencias inmunitarias provocadas por la aplicación de drogas inmunosupresoras o citostáticas, o por procesos patológicos tales como leucemia, enfermedad de Hodgkin o síndrome de inmunodeficiencia adquirida (SIDA), entre otros. En todos estos casos hay predisposición a la reactivación de una infección latente y la nueva diseminación del parásito puede resultar en una toxoplasmosis fatal, lo cual da un nuevo matiz de importancia a esta parasitosis.

\section{REFERENCIAS.}

1.- Kasper LH. Toxoplasma infection. En: Braunwald E, Fauci AS, Kasper DL, Hauser SL, Longo DL, Jameson JL, editores. Harrinson's Principles of Internal Medicine. 15 edition. New York: McGraw-Hill 2001.p. 1222-7.

2.- Acosta C, Pérez X, García R. Presencia de anticuerpos IgG anti-Toxoplasma gondii en embarazadas residentes en

\section{Revista Biomédica}




\section{Prevalencia de Acs-T.gondii IgG en donantes de sangre cubanos.}

Ciudad de la Habana. Rev Biomed 2001; 12: 250-4.

3.- González T, Bacallao J, García C, Molina JR. Prevalencia de anticuerpos anti-Toxoplasma gondii en una población de mujeres embarazadas en Cuba. Gac Méd Méx 1991; 131: 499-03.

4.- Collazo JE, López R, Ginorio D, Llamos R, Contreras R. Anticuerpos IgG anti-Toxoplasma gondii en pacientes con síntomas atribuíbles a toxoplasmosis. Biomed 1993; 13:17982.

5.- Martinez R, Bacallao R, Alberti E, Alfonso I. Prevalence of toxoplasmosis in pregnant women of the province of $\mathrm{La}$ Habana. Rev Inst Trop Sao Paulo 1994; 36: 445-50.

6.- Delgado G. A Cuban bibliography of toxoplasmosis II (1971-1980). Rev Cub Med Trop 1982; 34: 160-71.

7.- López R, Fano R, Contreras R, Font L. Anticuerpos IgG anti- Toxoplasma gondii en cubanos donantes de sangre. Rev Lat Amer Microbiol 1993; 35: 207-10.

8.- Thulliez Ph, Derouin F, Peyron F, Wallon M, Candolfi E. Toxoplasmosis and diagnostic. 1a ed. Madrid: Coimpress SA; 1996. p. 11.

9.- Sánchez A, Martín I, García S. Estudio de reactividad a Toxoplasma gondii en embarazadas de las provincias Ciudad de la Habana y Pinar del Río, Cuba. Bioquimia 2003; 111: 3-8.

10.- Góngora-Biachi RA, González-Martínez P, CastroSansores C, Álvarez-Moguel R, Pavía-Ruz N, Lara-Perera D, et al. Anticuerpos contra Toxoplasma gondii en pacientes con VIH en Yucatán. Rev Invest Clin 1998; 50: 419-22.

11.- Hirt J, Kaufer F, Carral L, Durlach R, Schmee E. Estudio comparativo de la prevalencia de la infección toxoplásmica en la ciudad de Buenos Aires entre los años 1967 y 1992 . VI Congreso Panamericano de Infectología, 26-29 Mayo de 1993, Viña del Mar, Chile.

12.- Bahía LM, Jones JL, Azevedo J, Alves CC, Orefice F, Dais DG. Highly endemic waterborne toxoplasmosis in North Rio de Janeiro state, Brasil. Emerg Infect Dis 2003; 9: 55-62.

13.- Orefice F, Bonfioli AA. Toxoplasmose. En: Orefice F, editor. Uveite clinica e cirugica. Rio de Janeiro: Editora Cultura Médica; 2000. p. 619-80.
14.- Arias L, Chinchilla M, Reyes L, Linder E. Seroepidemiology of transmission humans: posible transmisión routes in Costa Rica. Rev Biol Trop 1996; 44: 377-81.

15.- Wallace GD. Serologic and epidemiologic observations on toxoplasmosis on three Pacific atolls. Am J Epid 1969; 90 : 103-11.

16.- Machín R, Martínez R, Fachado A, Pividal J, Cruz R. Encuesta Nacional de Toxoplasmosis. Resumen: 50. Aniversario Inst. Med. Tropical “ Pedro Kourí 1988; 134.

17.- Acosta C, Pérez X, Herrera R, Solís RL, López R, Machín $\mathrm{R}$, et al. Evaluación clínica del UMELISA Toxoplasma. Congreso "Biotecnología Habana 92" 1992.

18.- Foulon W, Naessens A, Lauwer S. Impact of primary prevention on the incidence of toxoplasma during pregnancy. Obstet Gynecol 1988; 72: 363.

19.- Jackson MH, Hutchison WN. A seroepidemiological survey of toxoplasmosis in Scotland and England. Ann Trop Med Parasit 1987; 81: 360.

20.- Logar J, Novak-Antolic Z, Zore A, Cerar V, Likar M. Incidence of the congenital toxoplasmosis in the Republic of Slovenia. Scand J Infect Dis 1992; 24: 105-8.

21.- Remington JS, McLeod R, Desmonts G. Toxoplasmosis. En: Remington JS, Klein JO, editores. Infectious diseases of the fetus and newborn infants. 5th edition. Philadelphia: W.B. Saunders Company; 2001. p. 205-346. 